\title{
QTL mapping and genetic effect of chromosome segment substitution lines with excellent fiber quality from Gossypium hirsutum $\times$ Gossypium barbadense
}

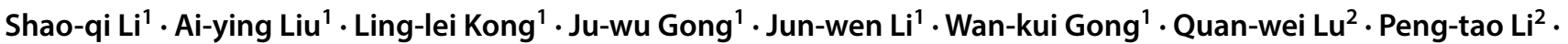 \\ Qun Ge ${ }^{1} \cdot$ Hai-hong Shang ${ }^{1} \cdot$ Xiang-hui Xiao ${ }^{1} \cdot$ Rui-xian Liu ${ }^{1} \cdot$ Qi Zhang $^{1} \cdot$ Yu-zhen Shi ${ }^{1} \cdot$ You-lu Yuan ${ }^{1}$
}

Received: 6 November 2018 / Accepted: 3 April 2019 / Published online: 27 April 2019

(c) The Author(s) 2019

\begin{abstract}
Chromosome segment substitution lines (CSSLs) are ideal materials for identifying genetic effects. In this study, CSSL MBI7561 with excellent fiber quality that was selected from $\mathrm{BC}_{4} \mathrm{~F}_{3: 5}$ of CCRI45 (Gossypium hirsutum) $\times$ Hail (Gossypium barbadense) was used to construct 3 secondary segregating populations with 2 generations $\left(\mathrm{BC}_{5} \mathrm{~F}_{2}\right.$ and $\left.\mathrm{BC}_{5} \mathrm{~F}_{2: 3}\right)$. Eighty-one polymorphic markers related to 33 chromosome introgressive segments on 18 chromosomes were finally screened using 2292 SSR markers which covered the whole tetraploid cotton genome. A total of 129 quantitative trait loci (QTL) associated with fiber quality (103) and yield-related traits (26) were detected on 17 chromosomes, explaining $0.85-30.35 \%$ of the phenotypic variation; 39 were stable (30.2\%), 53 were common (41.1\%), 76 were new (58.9\%), and 86 had favorable effects on the related traits. More QTL were distributed in the Dt subgenome than in the At subgenome. Twenty-five stable QTL clusters (with stable or common QTL) were detected on 22 chromosome introgressed segments. Finally, the 6 important chromosome introgressed segments (Seg-A02-1, Seg-A06-1, Seg-A07-2, Seg-A07-3, Seg-D07-3, and Seg-D06-2) were identified as candidate chromosome regions for fiber quality, which should be given more attention in future QTL fine mapping, gene cloning, and marker-assisted selection (MAS) breeding.
\end{abstract}

Keywords CSSLs $\cdot$ Chromosome introgressed segments · Fiber quality $\cdot$ QTL $\cdot$ Genetic effects

\section{Introduction}

Communicated by S. Hohmann.

Shao-qi Li and Ai-ying Liu have contributed equally to this work

Electronic supplementary material The online version of this article (https://doi.org/10.1007/s00438-019-01566-8) contains supplementary material, which is available to authorized users.

Yu-zhen Shi

shiyzmb@126.com

$\triangle$ You-lu Yuan

yuanyoulu@caas.cn

1 State Key Laboratory of Cotton Biology, Key Laboratory of Biologiacl and Genetic Breeding of Cotton, The Ministry of Agriculture, Institute of Cotton Research, Chinese Academy of Agricultural Science, Anyang 455000, Henan, China

2 School of Biotechnology and Food Engineering, Anyang Institute of Technology, Anyang 455000, Henan, China
As an allopolyploid cash crop, cotton is important for genetic research and provides the textile industry with the most important natural fiber raw material. Among cultivated cotton species, two tetraploid cottons, Upland cotton (Gossypium hirsutum L./G.h) with higher yield and Sea-island cotton (Gossypium barbadense L./G.b) with better fiber quality are the most widely cultivated in agricultural production (Ulloa et al. 2005; Zhang et al. 2009). Therefore, it is interesting to introgress the favorable genes for fiber quality from $G . b$ to $G . h$ to improve the fiber quality and yield simultaneously. However, introgression is very difficult for breeders to implement using conventional breeding, because all of the related traits are quantitative traits controlled by multiple genetic loci, and the fiber quality and yield-related traits are usually negatively correlated (Clement et al. 2012; Ma et al. 2014; Yu et al. 2016). Fortunately, with the rapid development of high-precision molecular marker technology and gene mapping, an increasing number of genetic maps 
and QTL have been identified (Jia et al. 2016; Kushanov et al. 2016).

After the first molecular genetics map of cotton was constructed (Reinisch et al. 1994), a wide variety of populations were used to perform QTL mapping, which predominantly consists of $\mathrm{F}_{2}$ (Brown et al. 2005; Wang et al. 2010; Yu et al. 2013), double haploid (DH) (Lu et al. 2014; Cai et al. 2015), backcross (BC) (Chen et al. 2012; Shang et al. 2016; Wang et al. 2016b), backcross inbred lines (BILs) (Pang et al. 2012; Nie et al. 2015; Zhang et al. 2015a), recombinant inbred lines (RILs) (Zhang et al. 2015c; Jamshed et al. 2016; Shang et al. 2016) and CSSLs (Liang et al. 2010; Li et al. 2016; Shi et al. 2016; Zhai et al. 2016). The complex genetic background in most populations makes it difficult to estimate the positions and effects of QTL. There are a few differences between CSSLs and recurrent parents, which is favorable for QTL mapping, genetic effect identification and gene cloning (Wang et al. 2016c; Lu et al. 2017). Therefore, more attention has been paid to the development and utilization of CSSLs in genetic research, although CSSLs are time consuming and costly to construct (Wan et al. 2008; Zhao et al. 2009).

The first CSSLs were constructed in tomatoes by Eshed and Zamir (1994). Subsequently, researchers launched a wide range of studies and applications of CSSLs in rice (Wan et al. 2004), corn (Li et al. 2014), wheat (Liu et al. 2006) and other crops. The first set of cotton CSSLs was developed with TM-1 as the recipient parent by Stelly et al. (2005), and was used to analyze genetic effects, as well as genetic relationships for fiber quality and yield component traits with substitution of one chromosome (Stelly et al. 2005). After this study, a series of studies were carried out on cotton CSSLs (Wang et al. 2008, 2016c Chen et al. 2012; Su et al. 2014; Lu et al. 2017). Yang et al. (2009) detected 51 QTLs using 116 CSSLs originating from CCRI45 (G.h) and Hai1 (G.b). Wang et al. (2012) indicated the inheritance of long staple fiber qualities using the CSSLs developed by TM-1 and Hai7124. Fu et al. (2013) detected 12 QTLs associated with fiber quality and yield using the CSSLs from TM-1 and Sub18.

Although CSSLs are effective in QTL mapping, there is less information for detecting genetic effects from introgressing chromosome segments from Island cotton into Upland cotton. A series of CSSLs were constructed through the hybridization of CCRI45 (G.h), CCRI36 (G.h) and Hail (G.b) by our team(Shi et al. 2008). Subsequently, a high-density genetic linkage map was constructed that contained 2292 SSR markers and covered $5115.16 \mathrm{cM}$ of the cotton AD genome (Shi et al. 2015), and many QTL were identified using populations with various generations $\left(\mathrm{BC}_{4} \mathrm{~F}_{1}, \mathrm{BC}_{4} \mathrm{~F}_{3}, \mathrm{BC}_{4} \mathrm{~F}_{3: 5}\right.$ and $\mathrm{BC}_{4} \mathrm{~F}_{4}$ ) (Yang et al. 2009; Ge et al. 2012; Ma 2014; Guo et al. 2015; Lan et al. 2015). The genetic effects and heterosis of yield and yield component traits were analyzed through hybridizing 10 CSSLs according to North Carolina Design II (Li et al. 2016). A total of 70 QTL and their genetic effects for fiber yield-related traits and 29 QTL for fiber quality traits on 13 chromosomes were detected using CSSLs $\left(\mathrm{BC}_{5} \mathrm{~F}_{3}, \mathrm{BC}_{5} \mathrm{~F}_{3: 4}\right.$ and $\left.\mathrm{BC}_{5} \mathrm{~F}_{3: 5}\right)$ (He 2014). Twenty two QTL associated with fiber quality and yield traits on seven chromosomes were detected in $\mathrm{F}_{2}$ and $\mathrm{F}_{2: 3}$ with two CSSLs of MBI9749 and MBI9915 as parents (Guo et al. 2018). A total of 24 QTLs for fiber quality and 11 QTLs for yield traits were detected in the three segregating generations of double-crossed $F_{1}$ and $F_{2}$ and $\mathrm{F}_{2: 3}$, which were constructed using four CSSLs as parents (MBI9804×MBI9855) $\times($ MBI9752×MBI9134) (Zhai et al. 2016). Eighteen QTL for fiber quality and 6 QTL for yieldrelated traits across 7 chromosomes were detected using $\mathrm{BC}_{6} \mathrm{~F}_{2}$ and $\mathrm{BC}_{6} \mathrm{~F}_{2: 3}$ with two parents of CCRI36 (recurrent parent) and MBI9915(CSSL) (Song et al. 2017).

In the present study, $\mathrm{BC}_{5} \mathrm{~F}_{2}$ and $\mathrm{BC}_{5} \mathrm{~F}_{2: 3}$ populations were constructed by hybridization of CCRI45 (recurrent parent) and MBI7561 $\left(\mathrm{BC}_{4} \mathrm{~F}_{3: 5}\right)$ with excellent and stable fiber quality, to evaluate the genetic effects of the introgressed segments by SSR markers. This study is expected to lay the foundation for future studies, such as genetic mechanism exploring, QTL fine mapping, gene cloning and MAS breeding applications.

\section{Materials and methods}

\section{Plant materials and population development}

MBI7561 as the female parent was selected from the CSSLs $\mathrm{BC}_{4} \mathrm{~F}_{3: 5}$ which was constructed by advanced backcross and selfing of combination of CCRI45 (G.h) and Hail (G.b). The recurrent parent CCRI45 was a glandular cotton cultivar widely grown with high yield and resistance to budworm (Ma 2014), which was developed by the Institute of Cotton Research of Chinese Academy of Agricultural Sciences (Shi et al. 2008, 2015). The donor parent Hail was a dominant glandless $G$. barbadense with excellent fiber quality. The fiber quality and yield component traits of MBI7561 were excellent and stable (Table 4).

We constructed $\mathrm{F}_{1}\left(\mathrm{BC}_{5} \mathrm{~F}_{1}\right)$ by backcrossing CCRI45 (male) and MBI7561 in Anyang in the summer of 2013. $\mathrm{BC}_{5} \mathrm{~F}_{1}$ was planted and self-crossing seeds $\left(\mathrm{BC}_{5} \mathrm{~F}_{2}\right)$ were harvested in Hainan province in the winter of 2013. In 2014 , a total of $310 \mathrm{BC}_{5} \mathrm{~F}_{2}$ (PopE1) individual plants were developed and fiber and seeds $\left(\mathrm{BC}_{5} \mathrm{~F}_{2: 3}\right)$ were collected from individual plants. Both $\mathrm{BC}_{5} \mathrm{~F}_{1}$ and $\mathrm{BC}_{5} \mathrm{~F}_{2}$ populations were planted in the Anyang experimental farm of the Institute of Cotton Research of Henan Province of China, with row length of $8 \mathrm{~m}$, row spacing of $0.8 \mathrm{~m}$, and plant spacing of $0.25 \mathrm{~m}$. In 2015 , a total of $253 \mathrm{BC}_{5} \mathrm{~F}_{2: 3}$ (PopE2) family 
lines were planted in one-row plot with a row length of $5 \mathrm{~m}$ on the Anyang experimental farm, and another 250 $\mathrm{BC}_{5} \mathrm{~F}_{2: 3}$ (PopE3) family lines were planted in two-narrowrow plots, with row length of $3 \mathrm{~m}$ and plant spacing of 0.12 $\mathrm{m}$ on the Alar experimental farm of the Institute of Cotton Research of Xinjiang Autonomous Region of China. The plastic-membrane covering technique and a wide/narrow row spacing pattern were used. The row spacing alternation was $0.2 \mathrm{~m}$ and $0.6 \mathrm{~m}$. In addition, there were 212 common $\mathrm{BC}_{5} \mathrm{~F}_{2: 3}$ lines in the two different environments.

\section{Investigation of phenotypic traits}

\section{Collection of phenotypic data}

In 2014, naturally opened bolls were collected from the $\mathrm{F}_{2}$ individual plants for phenotype evaluation, including boll weight (BW), lint percentage (LP), fiber length (FL), fiber strength (FS), fiber micronaire (FM), fiber uniformity (FU), and fiber elongation (FE). In 2015, 30 naturally opened bolls were harvested from the plot for $\mathrm{F}_{2: 3}$ lines. BW and LP were calculated using seed cotton weight, fiber weight, and boll number. The fiber quality traits were tested with HFT9000 using HVICC international calibration cotton samples in the Cotton Quality Supervision and Testing Center of the Ministry of Agriculture of China.

\section{Analysis of phenotypic traits}

The observed phenotypic data were analyzed using 3 softwares. BW, LP, and transgressive rate over the recurrent parent (TRORP) were calculated using EXCEL 2010. The SAS statistical software (version 8.1, SAS Institute, Cary $\mathrm{NC}$ ) was used to perform the descriptive statistical analysis of phenotypes. The statistical values included mean, maximum, minimum, standard deviation (SD), skewness, kurtosis, and coefficient of variation (CV). For ANOVA, correlation analysis and all significance tests were performed using the SPSS20.0 software (SPSS, Chicago, IL, USA). All correlation values were Pearson Correlation Coefficients. A higher FM value is not necessarily for indicative of better fiber quality, and the fineness of the fiber is evaluated by the FM value grading, (A level (best): [3.7,4.2]; B level (better): [3.5, 3.6] and[4.3, 4.9]; C level (bad): $[0,3.4]$ and $[5.0, \infty)$ ). The FM values of CCRI45 in all environments were in the $\mathrm{C}$ level, thus, we selected individuals in the A level and B level for the calculation of transgressive rate values, and the range of FM values of the selected individuals should be $\geq 3.5$ and $\leq 4.9$.

\section{Identification of genotypes}

\section{DNA extraction and marker detection}

The young leaves of $F_{2}$ individual plants and parents were sampled in 2014. Genomic DNA was extracted using the modified CTAB method(Paterson et al. 1993). The products of PCR amplification were isolated and identified by $8 \%$ non-denaturing vertical polyacrylamide gel electrophoresis. The DNA segments in the gel were visualized by the silver staining method (Zhang et al. 2000). In this study, we used 2292 SSR markers from the genetic linkage map with a total genetic length of $5115.16 \mathrm{cM}$ (Shi et al. 2015) to screen the recurrent parent of CCRI45, donor parent of Hai1, MBI7561 and $\mathrm{F}_{1}(\mathrm{MBI} 7561 * \mathrm{CCRI} 45)$. The polymorphic markers were used to identify genotypes of the $\mathrm{F}_{2}$ individual plants. The sequences of each primer were obtained from the Cotton Genome Database (www.cottongen.org) and synthesized by Bioethics Engineering (Shanghai) Co., Ltd.

\section{Analysis of genotypes}

Genotyping analysis of each sample and distribution analysis of chromosome introgressed segments were performed by GGT2.0 software (http://www.plantbreeding.wur.nl) (Van Berloo 2008), including the number, length and positions of introgressed segments, and the genetic background recovery rate of each sample. The nomenclature of segments was as follows: Seg + the serial number of the chromosome (AD) + the serial number of the cluster on the chromosome.

\section{Genetic effects analysis}

\section{QTL mapping}

The QTL IciMappingV4.1 (www.isbreeding.net/softw are/?type $=$ detail\&id $=18$ ) software was used to perform QTL mapping. A likelihood of odds (LOD) threshold of 2.5 was used to declare significant additive QTL. The resulting linkage map and QTL were drawn using MapChart2.2 software (Voorrips 2002). The QTL nomenclature was: $q+$ the English abbreviation of trait + the serial number of chromosome + the serial number of the QTL on the chromosome associated with the same trait + (the direction of the additive genetic effect). For example, $q F L-16-2(+)$ represents the second QTL associated with the FL on chromosome 16 with a positive additive genetic effect from the $G$. barbadense introgressing segments.

\section{QTL-cluster analysis}

The QTL cluster analysis was performed by Biomercator 4.2 software (Arcade et al. 2004). QTL were projected on the 
genetic map and QTL cluster analysis were performed for all traits. Four models were thus generated, and each had an Akaike information criterion (AIC) value. The model with the lowest AIC value was selected and used for the position identification of QTL clusters. The nomenclature of QTL cluster was: $\mathrm{Clu}+$ the English abbreviation of trait + the serial number of chromosome + the serial number of the cluster on the chromosome associated with the same trait.

\section{Results}

\section{Phenotypic performance of CSSLs populations}

The descriptive statistics of phenotypic data for fiber yield and fiber quality traits, as well as their recurrent parent CCRI45, are presented in Table 1 . In the three populations, the average performance of BW was smaller than that of CCRI45, with a significant difference in PopE3; the average performance of FU was higher than that of CCRI45, with a significant difference in PopE3; and the other traits were significantly better than those of CCRI45 except for LP in PopE2. The TRORP for BW was $17.52-32.83 \%$ and $53.62-99.26 \%$ for other traits. Overall, there were many transgressive separations for most of traits in the populations. All traits showed a continuous normal distribution in three populations (Table 1, Fig. S1), which was consistent with the characteristics of quantitative traits. Significant positive correlations were found among most traits (FL and FS/FE/FU, FS and FU/FE, FU and FE/FM/BW, and FM and $\mathrm{FE} / \mathrm{LP} / \mathrm{BW}$ ), whereas significant negative correlations were found between FS and FM, LP, and FE / FL in most populations (Table 2).

\section{Analysis of introgressive segments}

A total of 81 pairs of polymorphic markers were screened between the parents and 33 introgressive segments distributed on 18 chromosomes (Fig. 1). The genetic background recovery rate of MBI7561 was $95.60 \%$, and the proportion of homozygous introgressive segments $(138.11 \mathrm{cM}, 2.7 \%)$ was significantly higher than that of heterozygous segments (86.96 cM, 1.7\%). Four chromosomes [Chr15(D1), Ch25(D6), Chr07(A7), and Chr16(D7)] had more introgressive segments than others.

The 81 pairs of SSR markers were used to screen the genotype of the $\mathrm{BC}_{5} \mathrm{~F}_{2}$ population, and 6 pairs of markers did not show the polymorphism. The average rate of background

Table 1 Phenotypic performance of fiber quality and yield-related traits in three populations

\begin{tabular}{|c|c|c|c|c|c|c|c|c|c|c|c|c|}
\hline \multirow{2}{*}{ Environment } & \multirow{2}{*}{ Trait } & \multicolumn{2}{|c|}{ CCRI45 } & \multicolumn{9}{|c|}{ Population } \\
\hline & & Mean & SD & Gener. & Mean & Min. & Max. & SD & Ske. & Kur. & TRORP. (\%) & $\mathrm{CV}(\%)$ \\
\hline \multirow[t]{7}{*}{ PopE1 } & BW (g) & 5.63 & 1.02 & \multirow[t]{7}{*}{$\mathrm{BC}_{5} \mathrm{~F}_{2}$} & 5.30 & 2.48 & 9.37 & 0.88 & 0.36 & 0.84 & 32.83 & 16.53 \\
\hline & LP (\%) & 37.54 & 3.22 & & $39.90 * *$ & 25.52 & 52.69 & 2.76 & -0.28 & 1.55 & 66.46 & 6.91 \\
\hline & FL (mm) & 29.48 & 1.19 & & $30.77 * *$ & 25.84 & 35.29 & 1.20 & -0.21 & 0.55 & 86.39 & 3.90 \\
\hline & FS (cN/tex) & 28.02 & 1.38 & & $31.47 * *$ & 23.50 & 39.60 & 2.13 & -0.02 & 0.33 & 94.55 & 6.77 \\
\hline & FM (unit) & 4.83 & 0.56 & & $4.38 * *$ & 2.56 & 6.09 & 0.60 & -0.37 & -0.16 & 72.11 & 13.71 \\
\hline & $\mathrm{FU}(\%)$ & 83.98 & 1.52 & & 84.41 & 78.20 & 88.00 & 1.53 & -0.67 & 0.69 & 65.34 & 1.81 \\
\hline & $\mathrm{FE}(\%)$ & 6.79 & 0.06 & & $6.84 * *$ & 6.50 & 7.10 & 0.06 & -0.22 & 0.39 & 94.60 & 0.94 \\
\hline \multirow[t]{7}{*}{ PopE2 } & BW (g) & 5.38 & 0.26 & \multirow[t]{7}{*}{$\mathrm{BC}_{5} \mathrm{~F}_{2: 3}$} & 5.02 & 3.30 & 6.57 & 0.68 & -0.01 & -0.55 & 31.77 & 13.54 \\
\hline & $\mathrm{LP}(\%)$ & 36.74 & 3.34 & & 38.63 & 32.61 & 44.13 & 2.17 & -0.14 & -0.22 & 79.51 & 5.62 \\
\hline & $\mathrm{FL}(\mathrm{mm})$ & 28.57 & 1.65 & & $30.86^{* *}$ & 26.30 & 34.90 & 1.73 & -0.18 & -0.73 & 88.45 & 5.62 \\
\hline & FS (cN/tex) & 28.65 & 3.39 & & $34.94 * *$ & 28.00 & 44.00 & 2.78 & 0.22 & -0.07 & 99.26 & 7.94 \\
\hline & FM (unit) & 5.07 & 0.31 & & $4.58 * *$ & 3.00 & 5.70 & 0.47 & -0.32 & 0.24 & 77.70 & 10.35 \\
\hline & $\mathrm{FU}(\%)$ & 84.35 & 1.37 & & 85.26 & 82.10 & 87.90 & 1.25 & -0.33 & -0.39 & 77.23 & 1.47 \\
\hline & FE $(\%)$ & 6.80 & 0.14 & & $6.94 * *$ & 6.60 & 7.30 & 0.13 & 0.16 & -0.11 & 76.72 & 1.82 \\
\hline \multirow[t]{7}{*}{ PopE3 } & BW (g) & 5.74 & 0.16 & \multirow[t]{7}{*}{$\mathrm{BC}_{5} \mathrm{~F}_{2: 3}$} & $5.36 * *$ & 3.54 & 7.23 & 0.43 & 0.06 & 1.45 & 17.52 & 8.08 \\
\hline & LP (\%) & 38.20 & 1.45 & & $40.84 * *$ & 32.99 & 48.00 & 2.15 & -0.25 & 0.21 & 89.40 & 5.28 \\
\hline & FL (mm) & 28.07 & 0.91 & & $29.80 * *$ & 26.40 & 34.10 & 1.25 & 0.08 & 0.06 & 92.59 & 4.18 \\
\hline & FS (cN/tex) & 26.25 & 1.08 & & $28.96^{* *}$ & 24.40 & 35.00 & 1.83 & 0.20 & -0.06 & 94.18 & 6.31 \\
\hline & FM (unit) & 5.09 & 0.37 & & $4.80 * *$ & 3.40 & 5.80 & 0.43 & -0.34 & -0.02 & 60.67 & 8.95 \\
\hline & $\mathrm{FU}(\%)$ & 84.30 & 0.90 & & $85.24 * *$ & 82.00 & 88.10 & 1.16 & -0.25 & -0.15 & 78.23 & 1.36 \\
\hline & $\mathrm{FE}(\%)$ & 6.79 & 0.09 & & $6.88 * *$ & 6.50 & 7.20 & 0.10 & -0.13 & 0.34 & 91.18 & 1.48 \\
\hline
\end{tabular}

Sta statistic, $S E$ std. error, TRORP transgressive rate over the recurrent parent, $S k e$ skewness, Kur kurtosis, $F L$ fiber length, $F S$ fiber strength, $F M$ fiber micronaire, $F U$ fiber uniformity, $F E$ fiber elongation, $B W$ boll weight, $L P$ lint percentage 
Table 2 Correlation of phenotypic traits in the three populations

\begin{tabular}{|c|c|c|c|c|c|c|}
\hline & BW & LP & FL & FS & FM & FU \\
\hline \multicolumn{7}{|c|}{ PopE1 } \\
\hline LP & $-0.19 * *$ & & & & & \\
\hline FL & 0.07 & $-0.36^{* *}$ & & & & \\
\hline FS & -0.08 & -0.14 & $0.5^{* *}$ & & & \\
\hline FM & $0.47 * *$ & 0.04 & -0.05 & $-0.36^{* *}$ & & \\
\hline FU & $0.29 * *$ & $-0.19 * *$ & $0.32 * *$ & $0.20 * *$ & $0.37 * *$ & \\
\hline $\mathrm{FE}$ & $0.23 * *$ & $-0.19 * *$ & $0.46^{* *}$ & $0.35^{* *}$ & $0.21 * *$ & $0.43 * *$ \\
\hline \multicolumn{7}{|c|}{ PopE2 } \\
\hline LP & $0.33 * *$ & & & & & \\
\hline FL & $0.46 * *$ & $0.20 * *$ & & & & \\
\hline FS & 0.01 & -0.03 & $0.61 * *$ & & & \\
\hline FM & $0.57 * *$ & $0.39 * *$ & 0.11 & $-0.22 * *$ & & \\
\hline $\mathrm{FU}$ & $0.26 * *$ & $0.18^{* *}$ & $0.32 * *$ & $0.22 * *$ & $0.24 * *$ & \\
\hline $\mathrm{FE}$ & $0.50 * *$ & $0.29 * *$ & $0.79 * *$ & $0.55^{* *}$ & $0.29 * *$ & $0.37 * *$ \\
\hline \multicolumn{7}{|c|}{ PopE3 } \\
\hline LP & -0.05 & & & & & \\
\hline FL & -0.12 & $-0.38 * *$ & & & & \\
\hline FS & -0.13 & $-0.16^{*}$ & $0.66^{* *}$ & & & \\
\hline FM & $0.33 * *$ & $0.34 * *$ & $-0.49 * *$ & $-0.38 * *$ & & \\
\hline $\mathrm{FU}$ & -0.04 & $0.18^{*}$ & 0.13 & $0.25^{* *}$ & -0.05 & \\
\hline $\mathrm{FE}$ & 0.03 & $-0.16^{*}$ & $0.62 * *$ & $0.50 * *$ & -0.13 & $0.24 * *$ \\
\hline
\end{tabular}

$F L$ fiber length, $F S$ fiber strength, $F M$ fiber micronaire, $F U$ fiber uniformity, $F E$ fiber elongation, $B W$ boll weight, $L P$ lint percentage

*Significant correlation at the 0.05 level (2-tailed), $* *$ Significant correlation at the 0.01 level (2-tailed)
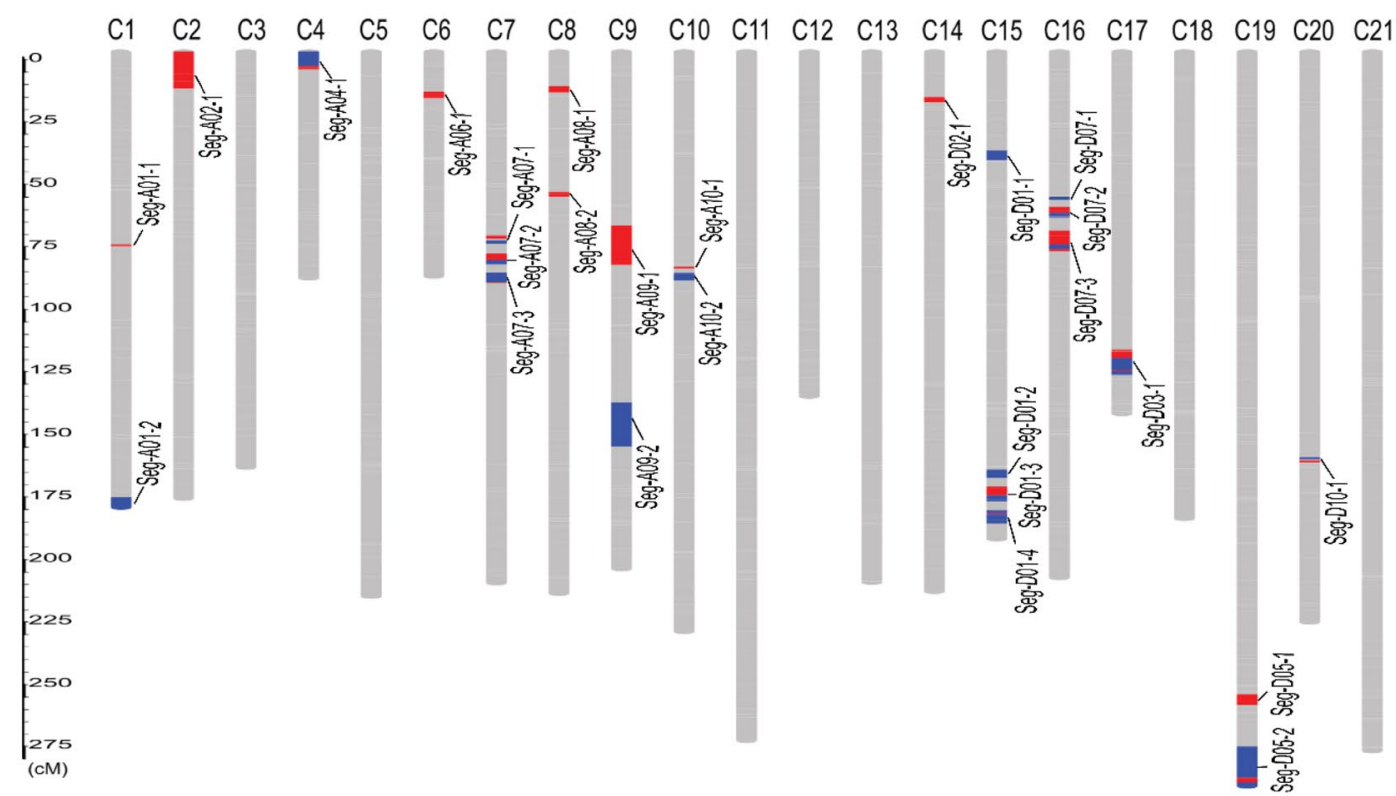

$\begin{array}{lllll}\mathrm{C} 22 & \mathrm{C} 23 & \mathrm{C} 24 & \mathrm{C} 25 & \mathrm{C} 26\end{array}$

Legend

: A

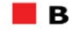

$\mathbf{H}$

Fig. 1 The graphical genotypes of MBI7561. Note A: genetic background (recurrent parent CCRI45); B: homozygous introgressive segments (donor parent Hai1); H: Heterozygous introgressive segments 
recovery in the $\mathrm{BC}_{5} \mathrm{~F}_{2}$ population was $97.95 \%$ and ranged from 97.3 to $99.2 \%$. The average rate of homozygous introgressive segments was $1.11 \%$ and ranged from 0 to $2 \%$. The average rate of heterozygous introgressive segments was $0.94 \%$ and ranged from 0.3 to $2.4 \%$.

\section{QTL mapping}

In total, 65 markers on 17 chromosomes were associated with the QTL of the seven traits, and 48 of these markers were associated with QTL in multiple populations. Nineteen markers located on 8 chromosomes existed in the At subgenome, and forty-six markers were located on 9 chromosomes in the Dt subgenome. Based on the method of inclusive composite interval mapping (ICIM), a total of 129 QTL were identified in 29 introgressive segments of 17 chromosomes in the three populations (Table 3, Table S1, Fig. S2), with each explaining $0.85 \%$ to $30.35 \%$ of the phenotypic variation (PVE). There were 103 and 26 QTL related to the five fiber quality traits and two yield component traits, respectively. Forty-one QTL were distributed in the At subgenome, and 88 were distributed in the Dt subgenome. In addition, there were 107 QTL distributed in 7 pairs of homologous chromosomes. Forty-five QTL (35\% of the total number) were detected in multiple environments, and 39 of them were stable. Eighty-six QTL showed positive additive effects, 36 showed negative additive effects, and seven showed unstable additive effects.

\section{Fiber length}

There were 21 QTL for FL on 11 chromosomes with the PVE ranging from $1.67 \%$ to $11.93 \% ; 7$ and 14 of these QTL were distributed in the At- and Dt subgenomes, respectively. Chr 16 and $\mathrm{Chr} 25$ were the top 2 chromosomes with the largest number of QTL. Sixteen QTL with additive effects from 0.06 to $0.75 \mathrm{~mm}$ indicated that Hai1 alleles increased FL, and four QTL with additive effects from -0.60 to $-0.22 \mathrm{~mm}$ indicated that CCRI45 alleles increased FL. The $q F L-16-3$ had unstable genetic effects, which could be detected in two environments, with additive effect of $-0.23 \mathrm{~mm}$ in PopE2 and $0.58 \mathrm{~mm}$ in PopE3. Three QTL ( $q F L-16-5, q F L-25-2$ and $q F L-25-3$ ) could be stably detected in more environments. The $q F L-25$ 2 linked to CGR5525 could explain $2.84 \%, 2.57 \%$, and $5.51 \%$ of the observed phenotypic variations with additive effects of $-0.57,-0.50$, and $-0.57 \mathrm{~mm}$ in PopE1, PopE2, and PopE3, respectively. The $q F L-16-5$ linked to NAU5408 and NAU3594 could explain 5.93\% and 9.60\% of the observed phenotypic variations in PopE1 and PopE3 with the additive effect of 0.23 and $0.29 \mathrm{~mm}$ in two generations, respectively. The $q F L-25-3$ linked to GH537 could explain $3.39 \%$ and $6.43 \%$ of the observed phenotypic variations in PopE1 and PopE3 with the additive effect of 0.22 and $0.50 \mathrm{~mm}$ in two generations, respectively.
Table 3 Distribution of QTL on chromosomes

\begin{tabular}{lcccccccc}
\hline & BW (g) & LP (\%) & FL (mm) & FS (cN.tex-1) & FM (unit) & FU (\%) & FE (\%) & Total \\
\hline Chr01(A1) & 0 & 1 & 0 & 1 & 1 & 1 & 1 & 5 \\
Chr02(A2) & 1 & 0 & 1 & 2 & 1 & 0 & 1 & 6 \\
Chr04(A4) & 1 & 0 & 1 & 0 & 0 & 0 & 0 & 3 \\
Chr06(A6) & 1 & 0 & 1 & 1 & 1 & 0 & 1 & 5 \\
Chr07(A7) & 1 & 1 & 2 & 3 & 1 & 1 & 2 & 11 \\
Chr08(A8) & 0 & 0 & 0 & 0 & 0 & 1 & 0 & 1 \\
Chr09(A9) & 0 & 1 & 1 & 1 & 0 & 1 & 2 & 6 \\
Chr10(A10) & 0 & 1 & 1 & 1 & 1 & 0 & 0 & 4 \\
Chr15(D1) & 1 & 4 & 2 & 3 & 1 & 4 & 5 & 20 \\
Chr16(D7) & 4 & 0 & 5 & 5 & 3 & 0 & 1 & 18 \\
Chr17(D3) & 0 & 0 & 1 & 2 & 1 & 2 & 0 & 6 \\
Chr19(D5) & 1 & 0 & 2 & 1 & 2 & 2 & 2 & 10 \\
Chr20(D10) & 0 & 0 & 0 & 0 & 1 & 0 & 0 & 1 \\
Chr22(D4) & 1 & 1 & 0 & 0 & 1 & 1 & 0 & 4 \\
Chr23(D9) & 0 & 1 & 0 & 1 & 0 & 0 & 0 & 2 \\
Chr24(D8) & 0 & 0 & 0 & 1 & 1 & 1 & 1 & 4 \\
Chr25(D6) & 3 & 2 & 4 & $\mathbf{5}$ & $\mathbf{3}$ & 2 & 4 & 23 \\
Total & 14 & 12 & 21 & 27 & 18 & 16 & 21 & 129 \\
\hline
\end{tabular}

$F L$ fiber length, $F S$ fiber strength, $F M$ fiber micronaire, $F U$ fiber uniformity, $F E$ fiber elongation, $B W$ boll weight, $L P$ lint percentage 


\section{Fiber strength}

There were 27 QTL for FS on 13 chromosomes with the PVE ranging from $0.85 \%$ to $13.19 \%$; 9 and 18 of them were distributed in the At- and Dt subgenomes, respectively. Chr16 and Chr25 were the first 2 chromosomes with the most QTL. Seventeen QTL with additive effects from 0.03 to $3.11 \mathrm{cN}^{-1} \mathrm{x}^{-1}$ indicated that Hail alleles increased FS, nine QTL with additive effects from -1.25 to -0.07 $\mathrm{cN}$ tex ${ }^{-1}$ indicated that CCRI45 alleles increased FS. The $q F S-17-1$ had unstable genetic effects, which could be detected in two environments, with additive effects of $0.35 \mathrm{cN} \mathrm{tex}^{-1}$ in PopE2 and $-0.07 \mathrm{cN}$ tex ${ }^{-1}$ in PopE3. Ten QTL ( $q F S-02-2, q F S-06-1, q F S-07-2, q F S-10-1$, $q F S-16-1, q F S-16-4, q F S-16-5, q F S-19-1, q F S-25-3$, and $q F S-25-4)$ could be stably detected in more environments. The qFS-16-1 linked to CGR6894a could explain 6.06\%, $7.25 \%$, and $7.38 \%$ of the observed phenotypic variations with the additive effect of $0.44,0.66$, and $0.42 \mathrm{cN} \mathrm{tex}^{-1}$ in PopE1, PopE2, and PopE3, respectively. The $q F S-16-5$ linked to NAU5408 and NAU3594 could explain 6.55\%, $7.42 \%$, and $10.92 \%$ of the observed phenotypic variations with additive effects of $0.66,0.78$, and $0.76 \mathrm{cN} \mathrm{tex}^{-1}$ in PopE1, PopE2, and PopE3, respectively. The qFS-253 linked to DPL0166a and SHIN-0885 could explain $13.09 \%, 8.16 \%$, and $7.91 \%$ of the observed phenotypic variations with additive effects of $0.04,3.11$, and $0.81 \mathrm{cN}$ tex $^{-1}$ in PopE1, PopE2, and PopE3, respectively. The $q F S$ 25-4 linked to CGR5201 and SHIN1131 could explain $11.95 \%, 6.95 \%$, and $4.90 \%$ of the observed phenotypic variations with additive effects of $0.89,0.81$, and $0.54 \mathrm{cN}$ tex $^{-1}$ in PopE1, PopE2, and PopE3, respectively. The $q F S$ 02-2 linked to DPL0450 and PGML04760 could explain $2.10 \%$ and $0.85 \%$ of the observed phenotypic variations in PopE2 and PopE3 with additive effects of 1.10 and $0.65 \mathrm{cN}$ tex $^{-1}$, respectively. The $q F S-06-1$ linked to DC40067 and DPL0918 could explain 3.99\% and 5.92\% of the observed phenotypic variations in PopE2 and PopE3 with additive effects of 1.48 and $1.16 \mathrm{cN}$ tex ${ }^{-1}$, respectively. The $q F S$ 07-2 linked to NAU2002 and CGR6381 could explain $5.85 \%$ and $10.38 \%$ of the observed phenotypic variations with additive effects of 0.58 and $0.78 \mathrm{cN} \mathrm{tex}{ }^{-1}$ in PopE1 and PopE3, respectively. The $q F S-10-1$ linked to DPL0468 could explain $5.03 \%$ and $5.95 \%$ of the observed phenotypic variations with additive effects of 0.66 and $0.48 \mathrm{cN}$ tex $^{-1}$ in PopE1 and PopE3, respectively. The qFS-16-4 linked to PGML02608 could explain $6.98 \%$ and $6.32 \%$ of the observed phenotypic variations with additive effect of 0.66 and $0.55 \mathrm{cN}$ tex ${ }^{-1}$ in PopE1 and PopE3, respectively. The $q F S-19-1$ linked to DC40425 and BNL3089 could explain $4.90 \%$ and $2.32 \%$ of the observed phenotypic variations with additive effects of 0.21 and $0.15 \mathrm{cN}^{-1}$ tex PopE1 and PopE2, respectively.

\section{Fiber micronaire}

A total of 18 QTL for FM on 13 chromosomes with the PVE ranging from 1.60 to $10.28 \%$ were identified; 5 and 13 of them were distributed in the At- and Dt subgenomes, respectively. Chr16 and Chr25 were the two most prominent chromosomes containing the largest number of QTL. Two QTL ( $q F M-16-1$ and $q F M-17-1$ ) with additive effects of 0.03 and 0.18 indicated that CCRI45 alleles decreased FM, and the remaining 16 QTL with additive effects from -0.52 to -0.06 indicated that Hai1 alleles decreased FM. Nine QTL ( $q F M-02-1, q F M-10-1, q F M-15-1, q F M-16-2, q F M-19-1$, $q F M-22-1, q F M-24-1, q F M-25-1$, and $q F M-25-2$ ) could be stably detected in more environments. The $q F M-02-1$ linked to PGML02861 and DPL0450 could explain 2.07\%, 9.24\%, and $6.25 \%$ of the observed phenotypic variations with additive effects of $-0.14,-0.52$, and -0.15 in PopE1, PopE2, and PopE3, respectively. The $q F M-15-1$ linked to NAU3177 could explain $2.35 \%, 3.22 \%$, and $4.73 \%$ of the observed phenotypic variations with additive effects of $-0.14,-0.08$ and - 0.16 in PopE1, PopE2 and PopE3, respectively. The $q F M$ 10-1 linked to DPL0468 could explain $4.56 \%$ and $3.56 \%$ of the observed phenotypic variations with additive effects of -0.17 and -0.13 in PopE1 and PopE3, respectively. The qFM-16-2 linked to HAU1836 and BNL2634 could explain $9.88 \%$ and $1.98 \%$ of the observed phenotypic variations with additive effect of -0.29 and -0.11 in PopE2 and PopE3, respectively. The $q F M-19-1$ linked to PGML01289 could explain $1.88 \%$ and $1.72 \%$ of the observed phenotypic variations with additive effects of -0.10 for both PopE2 and PopE3, respectively. The $q F M-22-1$ linked to JESPR230 and DPL0489 could explain 5.76\% and 3.28\% of the observed phenotypic variations in PopE2 and PopE3, respectively, with the additive effect of -0.08 and -0.15 . The $q F M-24$ 1 linked to NAU2914 could explain $1.87 \%$ and $1.60 \%$ of the observed phenotypic variations with additive effects of -0.09 and -0.08 in PopE1 and PopE3, respectively. The $q F M-25-1$ linked to DPL0166a and Gh537 could explain $2.05 \%$ and $6.22 \%$ of the observed phenotypic variations with additive effects of -0.06 and -0.25 in PopE1 and PopE3, respectively. The $q F M-25-2$ linked to BNL3806 and TMB 0313 could explain $10.28 \%$ and $5.91 \%$ of the observed phenotypic variations in PopE2 and PopE3, respectively, with additive effects of -0.18 and -0.09 .

\section{Fiber uniformity}

Sixteen QTL for FU were identified on 10 chromosomes with PVE ranging from $1.06 \%$ to $12.67 \%$; 4 and 12 of them were distributed in the At- and Dt subgenomes, respectively. Chr15 was the most prominent chromosome with the most QTL. Eleven QTL with additive effects from 0.01 to $0.65 \%$ indicated that Hai1 alleles increased FU. Two QTL 
( $q F U-19-1$ and $q F U-24-1$ ) with additive effects of $-0.49 \%$ and $-0.28 \%$ indicated that CCRI45 alleles increased FU. Three QTL ( $q F U-15-1, q F U-15-3$ and $q F U-15-4)$ could be detected in two environments, but had unstable genetic effects. Two QTL ( $q F U-01-1$ and $q F U-07-1$ ) could be stably detected in two environments. The $q F U-01-1$ linked to BNL2921 and NAU3901 could explain 8.44\% and 5.59\% of the observed phenotypic variations in PopE2 and PopE3, respectively, with additive effects of $0.37 \%$ and $0.12 \%$. The $q F U$-07- 1 linked to NAU1048 and CICR0226 could explain $1.89 \%$ and $1.82 \%$ of the observed phenotypic variations in PopE2 and PopE3, respectively, with the additive effect of $0.38 \%$ and $0.1 \%$.

\section{Fiber elongation}

There were 21 QTL for FE on 11 chromosomes with PVE ranging from $1.32 \%$ to $10.06 \% ; 8$ and 13 of these QTL were distributed in the At- and Dt subgenomes, respectively. Chr15 and Chr25 were the top 2 chromosomes with the largest number of QTL. Twelve QTL with additive effects from 0.001 to $0.05 \%$ indicated that Hail alleles increased FE, and eight QTL with additive effects from -0.14 to $-0.01 \%$ indicated that CCRI 45 alleles increased FE. The $q F E-02-1$ could be detected in PopE1 and PopE2, but had unstable genetic effects. Five QTL ( $q F E-06-1, q F E-16-1, q F E-19-2, q F E$ $25-2$, and $q F E-25-4$ ) could be stably detected in two environments. The $q F E-06-1$ linked to DC40067 and DPL0918 could explain $6.87 \%$ and $3.25 \%$ of the observed phenotypic variations in PopE2 and PopE3, respectively, with additive effects of $0.02 \%$ and $0.04 \%$. The $q F E-16-1$ linked to BNL2634 could explain $6.5 \%$ and $3.52 \%$ of the observed phenotypic variations in PopE1 and PopE2, respectively, with additive effects of $-0.04 \%$ and $-0.09 \%$. The $q F E-19$ 2 linked to DC40425 and HAU1400 could explain 7.13\% and $6.31 \%$ of the observed phenotypic variations in PopE2 and PopE3, respectively, with additive effects of $-0.01 \%$ and $-0.04 \%$. The $q F E-25-2$ linked to CICR0701 and Gh537 could explain $7.14 \%$ and $4.64 \%$ of the observed phenotypic variations in PopE1 and PopE3, respectively, with additive effects of $0.01 \%$ and $0.04 \%$. The $q F E-25-4$ linked to DPL0124 could explain $2.21 \%$ and $5.97 \%$ of the observed phenotypic variations in PopE2 and PopE3, respectively, with additive effects of $-0.05 \%$ and $-0.03 \%$.

\section{Boll weight}

A total of 14 QTL for BW were identified on 9 chromosomes with PVE ranging from 5.80 to $15.57 \% ; 4$ and 10 of them were distributed in the At- and Dt subgenomes, respectively. Chr 16 and Chr25 were the first 2 chromosomes with the most of QTL. Five QTL with additive effects from 0.01 to $0.28 \mathrm{~g}$ indicated that Hai1 alleles increased BW, and nine
QTL with additive effects from -0.57 to $-0.01 \mathrm{~g}$ indicated that CCRI45 alleles increased BW. Five QTL ( $q B W-06-1$, $q B W-07-1, q B W-16-2, q B W-16-4$ and $q B W-25-3)$ could be stably detected in more environments. The $q B W-06-1$ linked to DC40067 and DPL0918 could explain $6.61 \%, 6.72 \%$, and $6.07 \%$ of the observed phenotypic variations with additive effects of $-0.11,-0.22$, and $-0.08 \mathrm{~g}$ in the PopE1, PopE2, and PopE3, respectively. The $q B W-07-1$ linked to NAU2002 and NAU1085 could explain $8.61 \%$ and $6.98 \%$ of the observed phenotypic variations with additive effects of -0.36 and $-0.20 \mathrm{~g}$ in PopE1 and PopE2, respectively. The $q B W-16-2$ linked to HAU1836 and BNL2634 could explain $6.18 \%$ and $8.55 \%$ of the observed phenotypic variations with additive effects of -0.31 and -0.18 gin PopE1 and PopE2, respectively. The $q B W-16-4$ linked to NAU5408 and NAU3594 could explain $7.78 \%$ and $6.92 \%$ of the observed phenotypic variations with additive effects of -0.35 and -0.21 gin PopE1 and PopE2, respectively. The $q B W-25-3$ linked to BNL3806 and SHIN1131 could explain 7.85\% and $15.57 \%$ of the observed phenotypic variations in the PopE2 and PopE3, respectively, with additive effects of -0.22 and $-0.20 \mathrm{~g}$.

\section{Lint percentage}

Twelve QTL for LP were identified on 8 chromosomes with the PVE ranging from $3.39 \%$ to $30.35 \%, 4$ and 8 of them were distributed in At- and Dt subgenomes, respectively. Chr15 was the most prominent chromosome with the largest number of QTL. Nine QTL with the additive effect from 0.01 to $0.84 \%$ indicated that Hai1 alleles increased LP, and four QTL with the additive effect from -2.28 to $-0.52 \%$ indicated that CCRI45 alleles increased LP. Five QTL ( $q L P-09-1, q L P-15-1, q L P-15-2, q L P-15-3$ and $q L P-15-4)$ could be stably detected in more environments. The $q L P$ 09-1 linked to DPL0171 could explain 30.35\% and 4.92\% of the observed phenotypic variations with additive effects of $-2.28 \%$ and $-0.60 \%$ in PopE1 and PopE3, respectively. The $q L P-15-1$ linked to DPL0346a could explain $4.19 \%$ and $5.85 \%$ of the observed phenotypic variations with additive effects of $0.01 \%$ and $0.52 \%$ in PopE1 and PopE3 respectively. The $q L P-15-2$ linked to MUSS085 and SWU0280b could explain $5.80 \%$ and $11.47 \%$ of the observed phenotypic variations with additive effects of $0.78 \%$ and $0.84 \%$ in the PopE1 and PopE3, respectively. The $q L P-15-3$ linked to MUCS410 and HAU0059 could explain 5.55\%, 6.54\% and $5.42 \%$ of the observed phenotypic variations with additive effects of $0.01 \%, 0.25 \%$ and $0.72 \%$ in PopE1, PopE2 and PopE3, respectively. The $q L P-15-4$ linked to NAU5138 could explain $6.04 \%, 6.82 \%$ and $7.14 \%$ of the observed phenotypic variations with additive effects of $0.01 \%, 0.73 \%$ and $0.79 \%$ in PopE1, PopE2 and PopE3, respectively. 


\section{QTL cluster}

QTL clusters are chromosome regions that contain multiple QTL $(\geq 3)$ related to various traits (Rong et al. 2007). In the present study, 26 QTL clusters including 115 QTL were identified on 23 introgressive segments of 14 chromosomes (Table 3, Fig. S2); 8 and 19 of them were distributed in the At- and Dt subgenomes, respectively. The genetic length of the clusters varied from 1 to $22 \mathrm{cM}$ and was concentrated between 1 and $5 \mathrm{cM}$. There were more QTL clusters on Chr15, Chr16, and Chr25 than on the other chromosomes. Twenty-three QTL clusters had stable QTL with the same additive effect direction in more environments, and 11 of these QTL clusters had stable QTL for FS or FL. Two QTL clusters (Clu-16-5 and Clu-25-3) had stable QTL both for FS and FL. The Clu-16-5 on Chr16 at 78-80 cM had 4 QTL, the additive effects indicated that Hai1 alleles increased FL and FS, but decreased FM and BW. The Clu-25-3 on Chr25 at 25-28 cM had five QTL, and the additive effects indicated that Hai 1 alleles increased FL, FS, FU and LP, but decreased BW. Eight QTL clusters (Clu-02-1, Clu-06-1, Clu-07-2, Clu-10-1, Clu-16-1, Clu-16-4, Clu-19-2, and Clu-25-4) had

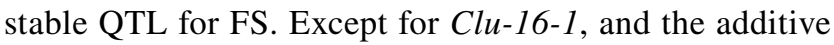
effects indicated that Hai1 alleles increased FL and FS, but decreased FM and BW. One QTL cluster $(\mathrm{Clu}-25-1)$ had stable QTL for FL, the additive effects indicated that Hail decreased FL and FS.

\section{Discussion}

\section{Selection of genetic linkage map and importance of CSSLs}

In total, 23,569 pairs of SSR markers distributed in the whole genome were used to screen for polymorphisms between CCRI36 (G.h) and Hai1 (G.b). A genetic linkage map with 2292 SSR loci on the 26 cotton chromosomes was developed from a $\mathrm{BC}_{1} \mathrm{~F}_{1}$ population of CCRI36 $\times$ Hail, covering the whole tetraploid cotton genome of $5115.16 \mathrm{cM}$ with an average distance of $2.23 \mathrm{cM}$ between adjacent markers (Shi et al. 2015). Genetic diversity is considered a critical issue in QTL mapping of complex traits. CSSLs have the potential to enrich the diversity of genetic background and uncover favorable alleles related to important fiber yield and quality traits(Ali et al. 2010; Wu et al. 2010; Tyagi et al. 2014). In addition, CSSLs are ideal materials for QTL mapping, genetic effects identifying and gene cloning(Yang et al. 2015), and are more convenient to study the minor and dominant effects of genes (Wan et al. 2004; He et al. 2015; Li et al. 2016; Qiao et al. 2016). In this study, the female parent MBI7561 was selected from a CSSL constructed by $G . h$ and $G . b$, which had stable and significant advantages for fiber quality compared with the recurrent parent of CCRI45, to produce $\mathrm{BC}_{5} \mathrm{~F}_{1}$ (Table 4). The length of introgressed segments from Hai1 for MBI7561 was $229.47 \mathrm{cM}$, of which homozygous introgressed segments were significantly longer than heterozygous introgressed segments. The total proportion of the introgressed segments for MBI7561 was small at the whole genome (4.40\%). However, the proportion of introgression in this study was relatively larger than that in the previous studies (Song et al. 2017; Guo et al. 2018). Thus, it is necessary to purify the genetic background using self-crossing and backcrossing. The diversity of the MBI7561 genotypes and the dominance of the phenotype were mutually beneficial, which showed that introgression segments had important effects on the phenotype. Moreover, this study laid an important foundation to construct a segregating population for identifying a large number of QTL related to fiber yield and quality. The performance of phenotypes (Tables 1, 2, Fig. S1) and genotypes (Fig. 1)
Table 4 Phenotypic performance of fiber quality and yield-related traits for parents

\begin{tabular}{lllllllll}
\hline Year & Parents & BW $(\mathrm{g})$ & LP $(\%)$ & FL $(\mathrm{mm})$ & FS (cN/tex) & FM (unit) & FU (\%) & FE (\%) \\
\hline 2012 & CCRI45 & 5.69 & 36.83 & 28.58 & 28.52 & 4.2 & 84.39 & 6.5 \\
& MBI7561 & $4.70^{* *}$ & 36.25 & $31.83^{* *}$ & $34.75^{* *}$ & $3.85^{* *}$ & $86.4^{* *}$ & 6.5 \\
& Hai1 & $3.18^{* *}$ & $32.31^{* *}$ & $32.18^{* *}$ & $37.7 * *$ & $4.81^{* *}$ & - & - \\
2013 & CCRI45 & 6.02 & 33.67 & 29.73 & 29.96 & 4.86 & 85.41 & 6.1 \\
& MBI7561 & $4.8^{* *}$ & $37.04 * *$ & $32.13^{* *}$ & $36.65^{* *}$ & $3.97 * *$ & $86.40^{*}$ & $5.9 * *$ \\
& Hai1 & $2.7 * *$ & $30.9^{* *}$ & $33.53^{* *}$ & $37.7 * *$ & $4.21^{* *}$ & $83.30^{* *}$ & $6.0^{* *}$ \\
2014 & CCRI45 & 5.63 & 37.54 & 29.48 & 28.02 & 4.83 & 83.98 & 6.8 \\
& MBI7561 & $4.99 * *$ & $39.56^{* *}$ & $31.58^{* *}$ & $33.59^{* *}$ & $4.15^{* *}$ & $84.86^{*}$ & 6.8 \\
2015 & CCRI45 & 5.38 & 36.74 & 28.57 & 28.65 & 5.07 & 84.35 & 6.8 \\
& MBI7561 & $4.72 * *$ & $39.28^{* *}$ & $31.5 * *$ & $32.6 * *$ & $4.40^{* *}$ & $85.00^{* *}$ & $7.0^{* *}$ \\
\hline
\end{tabular}

$F L$ fiber length, $F S$ fiber strength, $F M$ fiber micronaire, $F U$ fiber uniformity, $F E$ fiber elongation, $B W$ boll weight, $L P$ lint percentage

*Significant difference at the 0.05 level (2-tailed), **significant difference at the 0.01 level (2-tailed) 
in the $\mathrm{BC}_{5} \mathrm{~F}_{2}$ and $\mathrm{BC}_{5} \mathrm{~F}_{2: 3}$ populations showed a large number of superior materials and stable QTL in multiple environments.

\section{Common QTL shared with previous research}

A total of 129 QTL were identified in the present study, and 39 of them were stable (Table S1, Fig. S2). When QTL had the same linkage markers or confidence interval overlap between previous studies and our studies, we defined it as a common QTL interval (Zhang et al. 2016b). Fifty three common QTL detected in the present study were reported in the previous researches (Zhang et al. 2008, 2015b, c, 2016a, b; Liu 2009; Qin et al. 2009; Yang et al. 2009; Liang et al. 2010; Jia et al. 2011; Zhang 2012; Wang et al. 2013, 2016a, b; He 2014; Ma 2014; Cao et al. 2015; Guo et al. 2015; Nie et al. 2015; Rong et al. 2015; You 2015; Jamshed et al. 2016; Ademe et al. 2017; Li 2017; Song et al. 2017; Guo et al. 2018), and 15 of them were stable (Table S3). The remaining 76 of the 129 QTL were identified for the first time, and 24 of the 39 stable QTL $(q F L-16-5(+), q F L-25-2(-)$, $q F S-02-2(+), q F S-16-1(+), q F S-16-4(+), q F S-16-5(+)$, $q F S-19-1(+), q F M-02-1(-), q F M-10-1(-), q F M-15-1(-)$, $q F M-16-2(-), q F M-19-1(-), q F U-01-1(+), q F E-06-1(+)$, $q F E-16-1(-), q F E-19-2(-), q F E-25-4(-), q B W-07-1(-)$, $q B W-16-2(-), q B W-16-4(-), q B W-25-3(-), q L P-09-1(-)$, $q L P-15-2(+)$ and $q L P-15-4(+))$ were newly identified stable QTL in the present study. Both stable QTL and common QTL were suggestive of the stabilities of the genetic effects (Zhai et al. 2016). Therefore, these 77 stable or common QTL were very important for marker assisted breeding and exploration of genetic mechanisms.

\section{QTL clusters with common and stable QTL}

QTL clusters are common phenomena in cotton (Said et al. 2015; Wang et al. 2015; Zhai et al. 2016; Song et al. 2017). A large number of QTL were enriched in these hotspots, which indicated that these chromosome segments might contain pleiotropic or linked genes related to different traits (Abdelraheem et al. 2017). These clustered QTL may belong to the same genetic factor group contributing to the complex network of fiber development and affecting the multiple fiber traits(Lacape et al. 2010). QTL clusters allow cotton breeders to focus their efforts on regions with pleiotropic or linked loci. In the present study, a total of 26 QTL clusters were identified; 23 of them had stable QTL and 22 of them had 49 common QTL (Table S2, Table S3) (Zhang et al. 2008, 2015b, c, 2016a, b; Liu 2009; Qin et al. 2009; Yang et al. 2009; Liang et al. 2010; Jia et al. 2011; Zhang 2012; Wang et al. 2013; He 2014; Ma 2014; Cao et al. 2015; Guo et al. 2015; Nie et al. 2015; Rong et al. 2015; You 2015; Jamshed et al. 2016; Wang et al. 2016a, b; Ademe et al. 2017; Li
2017; Song et al. 2017; Guo et al. 2018). The remaining four clusters (Clu-02-1, Clu-04-1, Clu-16-4 and Clu-165) were new. Twenty-five QTL clusters contained the 77 stable or common QTL with stable genetic effects, which were regarded as stable QTL clusters. There were 19 stable QTL clusters for FL or FS, 7 (Clu-07-1, Clu-07-2, Clu-161, Clu-16-5, Clu-19-2, Clu-25-3 and Clu-25-4) of them for FL and FS, 5 (Clu-15-3, Clu-16-2, Clu-16-3, Clu-17-1 and $\mathrm{Clu}-25-1)$ of them mainly for FL, and 7 (Clu-02-1, Clu-061, Clu-10-1, Clu-15-1, Clu-15-4, Clu-16-4 and Clu-24-1) of them mainly for FS.

Fifteen of the 19 stable QTL clusters had common QTL for FS or FL. Four QTL clusters (Clu-07-1, Clu-07-2, Clu25-3 and Clu-25-4) had common QTL for both FS and FL, and the additive effects indicated most of Hai1 alleles increased FL, FS and LP, but decreased FM and BW. Six QTL clusters (Clu-15-3, Clu-16-1, Clu-16-2, Clu-16-3, Clu17-1 and Clu-19-2) had common QTL for FL. Except for Clu-15-3 and Clu-16-3, the additive effects of the others indicated Hai1 alleles increased FL and FS. Five QTL clusters (Clu-06-1, Clu-10-1, Clu-15-1, Clu-15-4 and Clu-24-1) had common QTL for FS. The additive effects of $\mathrm{Clu}-15$ 1, Clu-15-4 and Clu-24-1 indicated that most of the Hai1 alleles increased FL and FS.

Three (Clu-02-1, Clu-16-4 and Clu-16-5) of the 4 new QTL clusters were new stable QTL clusters. Their additive effects were similar to the effects of the 4 QTL clusters which had common QTL for both FS and FL.

\section{Genetic effects of the important chromosome introgressed segments}

Analyzing the genetic effects of the chromosome segments is necessary to develop a breeding strategy with precise direction in MAS (Zhai et al. 2016; Song et al. 2017), and to explore genetic mechanisms. In this study, the genetic effects of 29 chromosome introgressed segments were identified for fiber quality and yield-related traits. The genetic effects of 23 chromosome introgressed segments were identical to those of the QTL clusters associated with them. Four segments (Seg-A08-2, Seg-A09-1, Seg-D09-1, and Seg-D06-3) had stable genetic effects without any QTL clusters (Table S2).

A total of 13 chromosome introgressed segments were important for fiber quality (10) and LP (4) improvement on the 7 chromosomes [Chr15(D1), Chr02(A2), Chr19(D5), Chr06(A6)-Ch25(D6), Chr07(A7)-Chr16(D7)]. The additive effects of $\mathrm{Seg}$-D06-2 with 3 stable QTL clusters (with stable or common QTL) on Chr25(D6) at 23-33 cM indicated that most of the introgressed Hail alleles stably increased FL, FS, FU and FE, but stably decreased FM. The additive effects of 9 segments (Seg-A07-1, Seg-A07-2, Seg-A07-3, Seg-A02-1, Seg-A06-1, Seg-A10-2, Seg-D07-1, Seg-D07-3, and Seg-D05-2), with single stable QTL cluster 
(with stable or common QTL), indicated that most of the introgressed Hai1 alleles stably increased FL and FS, but stably decreased FM (except for Seg-D07-1). In addition, the additive effects of 4 important chromosome introgressed segments (Seg-A07-1, Seg-D01-2, Seg-D01-3, and Seg-D014) with single-stable QTL cluster (with stable or common QTL) indicated that the introgressed Hail alleles stably increased LP.

\section{Distribution of important chromosome introgressed segments for excellent lines}

To further verify which of the 13 important chromosome introgressed segments had major genetic effects, we analyzed the distribution of introgressed segments for 5 excellent lines with the best comprehensive phenotypes of FL, FS, FM, and LP in multiple populations (Table 5, Fig. 2).
There were 6 common important chromosome introgressed segments (Seg-A02-1, Seg-A06-1, Seg-A07-2, Seg-A07-3, Seg-D07-3 and Seg-D06-2) in all five lines, and the additive effects for the related QTL indicated that the introgressed Hail alleles stably improved FL, FS and FM. There were three important chromosome introgressed segments (SegD01-2, Seg-D01-3 and Seg-D01-4), with stable genetic effects for LP, enriched in the region (169-189 cM) on Chr15(D1). The introgressed region was detected in all 5 excellent lines. The distribution of the important chromosome introgressed segments (or region) in excellent lines further confirmed that further research should be focused on the 7 common important chromosome introgressed segments(or region) for fine mapping, genetic mechanisms exploring, and MAS breeding applications.

In conclusion, developing CSSLs is an effective method for identifying genetic effects. We selected the CSSLs

Table 5 Phenotypics and introgressed segments distribution of 5 lines with excellent fiber quality

Ind. ID Phenotype value of fiber quality in three environments

Chromosome introgressed segments

Fiber length (mm)

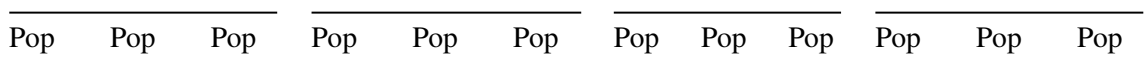

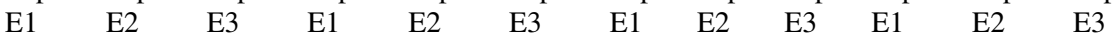

\begin{tabular}{llllllllllllllll}
\hline $288-02$ & 34.58 & 31.30 & 32.50 & 34.70 & 36.70 & 32.70 & 4.51 & 4.10 & 5.20 & 41.50 & 36.20 & 39.90 & 14 & 106.00 & 2.07 \\
$291-06$ & 35.29 & 31.20 & 34.10 & 37.90 & 36.70 & 33.40 & 3.90 & 3.80 & 3.90 & 38.18 & 35.72 & 39.91 & 14 & 100.00 & 1.95 \\
$291-19$ & 32.62 & 32.60 & 31.20 & 35.90 & 40.50 & 30.90 & 3.83 & 4.30 & 4.80 & 34.86 & 36.01 & 41.47 & 13 & 104.00 & 2.03 \\
$292-05$ & 34.43 & 33.10 & 33.10 & 39.30 & 38.40 & 32.40 & 3.24 & 4.00 & 5.10 & 33.45 & 35.45 & 38.86 & 14 & 107.00 & 2.09 \\
$243-04$ & 30.06 & 33.90 & 31.00 & 31.70 & 39.40 & 33.30 & 3.95 & 3.90 & 3.80 & 37.17 & 37.31 & 38.61 & 11 & 71.00 & 1.39 \\
MBI7561 & 31.58 & 31.50 & 31.01 & 33.59 & 32.60 & 30.40 & 4.15 & 4.40 & 4.42 & 39.56 & 39.28 & 40.80 & 33 & 127.00 & 2.48 \\
CCRI45 & 29.48 & 28.57 & 28.07 & 28.02 & 28.65 & 26.25 & 4.83 & 5.07 & 5.09 & 37.54 & 36.74 & 38.20 & - & - & - \\
\hline
\end{tabular}

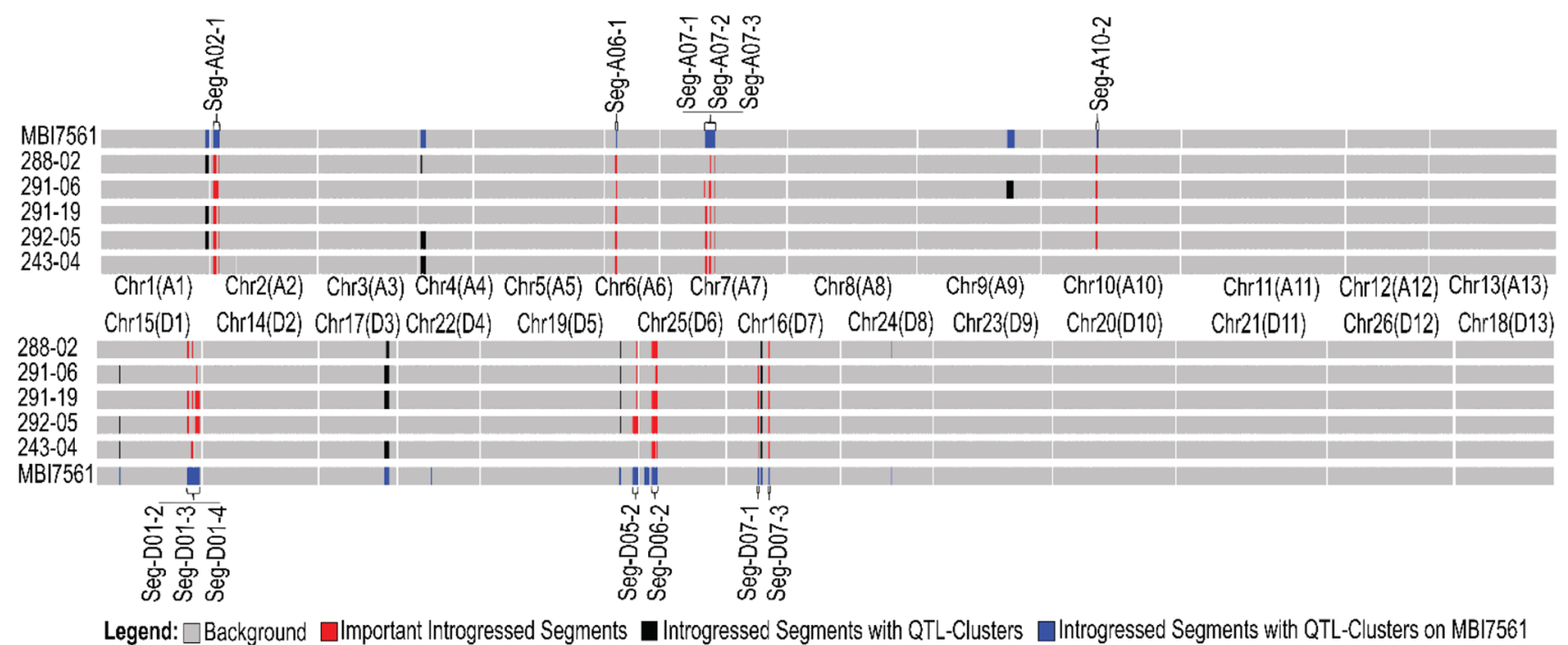

Fig. 2 Genotype distribution of individuals with excellent fiber quality 
containing 33 chromosome introgression segments as a parent to construct the three segregated populations. A total of 129 QTL associated with fiber quality (103) and yield-related traits (26) were detected on 17 chromosomes, explaining $0.85-30.35 \%$ of the phenotypic variation, 39 were stable, 53 were common, 76 were new, and 86 had favorable effects on the related traits. More QTL were distributed in the Dt subgenome than in the At subgenome. Twenty-five stable QTL clusters (with stable or common QTL) were detected on 22 chromosome introgressed segments. Finally, the 6 important chromosome introgressed segments (Seg-A02-1, Seg-A06-1, Seg-A07-2, Seg-A07-3, Seg-D07-3 and Seg-D06-2) were identified as candidate chromosome regions for fiber quality, which should be given more attention in future QTL fine mapping, gene cloning, and MAS breeding.

Acknowledgements This study was funded by the National Key R \& D Program for Crop Breeding (2016YFD0100306), the National Natural Science Foundation of China (31101188) and the Agricultural Science and Technology Innovation Program for CAAS (CAASASTIP-ICRCAAS). Thanks to the Quantitative Genetics Group of CAAS (Beijing, China) providing the software ICIMapping and help in QTL identification.

\section{Compliance with ethical standards}

Conflict of interest The authors declare that they have no conflict of interest.

Ethical approval This article does not contain any studies with human participants or animals performed by any of the authors.

Informed consent Informed consent was obtained from all individual participants included in the study.

Open Access This article is distributed under the terms of the Creative Commons Attribution 4.0 International License (http://creativeco mmons.org/licenses/by/4.0/), which permits unrestricted use, distribution, and reproduction in any medium, provided you give appropriate credit to the original author(s) and the source, provide a link to the Creative Commons license, and indicate if changes were made.

\section{References}

Abdelraheem A, Liu F, Song M, Zhang JF (2017) A meta-analysis of quantitative trait loci for abiotic and biotic stress resistance in tetraploid cotton. Mol Genet Genomics 292:1221-1235

Ademe MS, He S, Pan Z, Sun J, Wang Q, Qin H, Liu J, Liu H, Yang J, Xu D, Yang J, Ma Z, Zhang J, Li Z, Cai Z, Zhang X, Zhang X, Huang A, Yi X, Zhou G, Li L, Zhu H, Pang B, Wang L, Jia Y, Du $X$ (2017) Association mapping analysis of fiber yield and quality traits in Upland cotton (Gossypium hirsutum L.). Mol Genet Genomics 292:1267-1280

Ali ML, Sanchez PL, Yu SB, Lorieux M, Eizenga GC (2010) Chromosome segment substitution lines: a powerful tool for the introgression of valuable genes from Oryza wild species into cultivated rice (O. sativa). Rice 3:218-234

Arcade A, Labourdette A, Falque M, Mangin B, Chardon F, Charcosset A, Joets J (2004) BioMercator: integrating genetic maps and QTL towards discovery of candidate genes. Bioinformatics 20:2324-2326

Brown JS, Schnell RJ, Motamayor JC, Lopes U, Kuhn DN, Borrone JW (2005) Resistance gene mapping for witches' broom disease in Theobroma cacao $\mathrm{L}$. in an $\mathrm{F}_{2}$ population using SSR markers and candidate genes. J Am Soc Hortic Sci 130:366-373

Cai G, Yang Q, Yi B, Fan C, Zhang C, Edwards D, Batley J, Zhou Y (2015) A bi-filtering method for processing single nucleotide polymorphism array data improves the quality of genetic map and accuracy of quantitative trait locus mapping in doubled haploid populations of polyploid Brassica napus. BMC Genomics. https://doi.org/10.1186/s12864-015-1559-4

Cao Z, Zhu X, Chen H, Zhang T (2015) Fine mapping of clustered quantitative trait loci for fiber quality on chromosome 7 using a Gossypium barbadense introgressed line. Mol Breed. https:// doi.org/10.1007/s11032-015-0393-3

Chen X, Guo W, Liu B, Zhang Y, Song X, Cheng Y, Zhang L, Zhang $\mathrm{T}$ (2012) Molecular mechanisms of fiber differential development between $G$. barbadense and $G$. hirsutum revealed by genetical genomics. PLoS ONE 7:e30056

Clement JD, Constable GA, Stiller WN, Liu SM (2012) Negative associations still exist between yield and fibre quality in cotton breeding programs in Australia and USA. Field Crops Res 128:1-7

Eshed Y, Zamir D (1994) A genomic library of Lycopersicon pennellii in L.esculentum: a tool for fine mapping of genes. Euphytica 79:175-179

Fu Y, Yuan D, Hu W, Cai C, Guo W (2013) Development of Gossypium barbadense chromosome 18 segment substitution lines in the genetic standard line TM-1 of Gossypium hirsutum and mapping of QTLs related to agronomic traits. Acta Agron Sin 39:21-28

Ge R, Lan M, Shi Y, Li J, Liu A, Wang T, Yuan Y (2012) Correlation and path coefficient analysis of main agronomic characters in $\mathrm{BC}_{4} \mathrm{~F}_{3}$ and $\mathrm{BC}_{4} \mathrm{~F}_{4}$ generations from Gossypium hirsutum $\mathrm{L}$. $\times$ Gossypium barbadense L. Chin Agric Sci Bull 28:127-130

Guo L, Shi Y, Li J, Gong J, Liu A, Shang H, Gong W, Chen T, Ge Q, Sun J, Yuan Y (2015) Mapping QTL of fiber yield and quality traits in $\mathrm{F}_{2}$ populations of chromosome segment substitution lines from Gossypium hirsutum $\times$ Gossypium barbadense. Cotton Sci 27:550-560

Guo L, Shi Y, Gong J, Liu A, Tan Y, Gong W, Li J, Chen T, Shang H, Ge Q, Lu Q, Sun J, Yuan Y (2018) Genetic analysis of the fiber quality and yield traits in G. hirsutum background using chromosome segments substitution lines (CSSLs) from Gossypium barbadense. Euphytica 214:82. https://doi.org/10.1007/s1068 1-018-2158-7

He R (2014) The evaluation and identifying QTL of chromosome segment substitution lines $\left(\mathrm{BC}_{5} \mathrm{~F}_{3}, \mathrm{BC}_{5} \mathrm{~F}_{3: 4}, \mathrm{BC}_{5} \mathrm{~F}_{3: 5}\right)$ in CCRI36 background of Gossypium hirsutum L. [D], Chinese Academy of Agricultural Sciences cnki.net (S562, in Chinese)

He Q, Yang H, Xiang S, Tian D, Wang W, Zhao T, Gai J (2015) Fine mapping of the genetic locus $L 1$ conferring black pods using a chromosome segment substitution line population of soybean. Plant Breed 134:437-445

Jamshed M, Jia F, Gong J, Palanga KK, Shi Y, Li J, Shang H, Liu A, Chen T, Zhang Z, Cai J, Ge Q, Liu Z, Lu Q, Deng X, Tan Y, Or Rashid H, Sarfraz Z, Hassan M, Gong W, Yuan Y (2016) Identification of stable quantitative trait loci (QTLs) for fiber quality traits across multiple environments in Gossypium hirsutum recombinant inbred line population. BMC Genomics. https://doi.org/10.1186/ s12864-016-2560-2 
Jia F, Sun F, Li J, Liu A, Shi Y, Gong J, Shang H, Gong W, Wang T, Liu Z, Yuan Y (2011) Identification of QTL for boll weight and lint percentage of Upland cotton RIL population in multiple environments. Mol Plant Breed 9:318-326

Jia X, Pang C, Wei H, Wang H, Ma Q, Yang J, Cheng S, Su J, Fan S, Song M, Wusiman N, Yu S (2016) High-density linkage map construction and QTL analysis for earliness-related traits in Gossypium hirsutum L. BMC Genomics. https://doi.org/10.1186/ s12864-016-3269-y

Kushanov FN, Pepper AE, Yu JZ, Buriev ZT, Shermatov SE, Saha S, Ulloa M, Jenkins JN, Abdukarimov A, Abdurakhmonov IY (2016) Development, genetic mapping and QTL association of cotton $P H Y A, P H Y B$, and $H Y 5$-specific CAPS and dCAPS markers. BMC Genet. https://doi.org/10.1186/s12863-016-0448-4

Lacape JM, Llewellyn D, Jacobs J, Arioli T, Becker D, Calhoun S, Al-Ghazi Y, Liu S, Palai O, Georges S, Giband M, de Assuncao H, Augusto P, Barroso V, Claverie M, Gawryziak G, Jean J, Vialle M, Viot C (2010) Meta-analysis of cotton fiber quality QTLs across diverse environments in a Gossypium hirsutum $\times$ G. Barbadense RIL population. BMC Plant Biol. https://doi. org/10.1186/1471-2229-10-132

Lan M, Shi Y, Ge R, Li J, Gong J, Liu A, Shang H, Gong W, Wang T, Yuan Y (2015) Evaluation of fiber yield and quality traits of chromosome segment substitution lines population $\left(\mathrm{BC}_{4} \mathrm{~F}_{3}\right.$ and $\mathrm{BC}_{4} \mathrm{~F}_{3: 4}$ ) from Gossypium hirsutum $\times$ Gossypium barbadense. $\mathrm{J}$ Plant Genet Resour 16:257-263

Li B (2017) Genetic analysis and mapping of major QTL on yield and fiber quality related traits in cotton chromosome segment substitution lines. [D], Chinese Academy of Agricultural Sciences cnki. net (S562, in Chinese)

Li F, Jia H, Liu L, Zhang C, Liu Z, Zhang Z (2014) Quantitative trait loci mapping for kernel row number using chromosome segment substitution lines in maize. Genet Mol Res 13:1707-1716

Li B, Shi Y, Gong J, Li J, Liu A, Shang H, Gong W, Chen T, Ge Q, Jia C, Lei Y, Hu Y, Yuan Y (2016) Genetic effects and heterosis of yield and yield component traits based on Gossypium barbadense chromosome segment substitution lines in two Gossypium hirsutum backgrounds. PLoS ONE 11:e0157978

Liang Y, Jia Y, Li A, Zhang B, Liu G, Li J, Shi Y, Li J, Liu A, Gong J, Wang T, Shang H, Gong W, Yuan Y (2010) Phenotyping traits related to yield and quality of $\mathrm{BC}_{5} \mathrm{~F}_{2}$ substitution lines in cotton and their QTL mapping. Mol Plant Breed 8:221-230

Liu G (2009) Development and morphology of cotton fiber and locating the elite QTL alleles of G.barbadense L. fiber. [D], Hunan Agricultural University cnki.net (S562, in Chinese)

Liu SB, Zhou RG, Dong YC, Li P, Jia JZ (2006) Development, utilization of introgression lines using a synthetic wheat as donor. Theor Appl Genet 112:1360-1373

Lu L, Wei Q, Wang F, Liu G, Qin D, Guan P, Ni Z, Yao Y, Sun Q, Peng H (2014) QTL mapping for plant height and internode lengths in Nongda3338 $\times$ Jingdong6 DH populations of wheat (Triticum aestivum L.). J China Agric Univ 19:1-8

Lu Q, Shi Y, Xiao X, Li P, Gong J, Gong W, Liu A, Shang H, Li J, Ge Q, Song W, Li S, Zhang Z, Rashid MHO, Peng R, Yuan Y, Huang J (2017) Transcriptome analysis suggests that chromosome introgression fragments from Sea Island cotton (Gossypium barbadense) increase fiber strength in Upland cotton (Gossypium hirsutum). G3 7:3469-3479

Ma L (2014) The evaluation and QTL identifying of chromosome segment substitution lines in advanced backcross of Gossypium hirsutum $\times$ G.barbadense. [D], Chinese Academy of Agricultural Sciences cnki.net (S562, in Chinese)

Ma Q, Su J, Chen H, Deng F (2014) Research progress of cotton molecular marker-assisted breeding. Guangdong Agric Sci 41:138-143
Nie X, Tu J, Wang B, Zhou X, Lin Z (2015) A BIL population derived from $G$. hirsutum and $G$. barbadense provides a resource for cotton genetics and breeding. PLoS ONE 10:e0141064

Pang MX, Percy RG, Hughs SE, Jones DC, Zhang JF (2012) Identification of genes that were differentially expressed and associated with fiber yield and quality using cDNA-AFLP and a backcross inbred line population. Mol Breed 30:975-985

Paterson AH, Brubaker CL, Wendel JF (1993) A rapid method for extraction of cotton (Gossypium spp.) genomic DNA suitable for RFLP or PCR analysis. Plant Mol Biol Report 11:122-127

Qiao W, Qi L, Cheng Z, Su L, Li J, Sun Y, Ren J, Zheng X, Yang Q (2016) Development and characterization of chromosome segment substitution lines derived from Oryza rufipogon in the genetic background of $O$. sativa spp. indica cultivar 9311. BMC Genomics 17:89. https://doi.org/10.1186/s12864-016-2987-5

Qin Y-s, Ye W-x, Liu R-z, Zhang T-z, Guo W-z (2009) QTL mapping for fiber quality properties in Upland cotton (Gossypium hirsutum L.). Sci Agric Sin 42:4145-4154

Reinisch AJ, Dong JM, Brubaker CL, Stelly DM, Wendel JF, Paterson AH (1994) A detailed RFLP map of cotton, Gossypium hirsutum $\times$ Gossypium barbadense: chromosome organization and evolution in a disomic polyploid genome. Genetics 138:829-847

Rong J, Feltus EA, Waghmare VN, Pierce GJ, Chee PW, Draye X, Saranga Y, Wright RJ, Wilkins TA, May OL, Smith CW, Gannaway JR, Wendel JR, Paterson AH (2007) Meta-analysis of polyploid cotton QTL shows unequal contributions of subgenomes to a complex network of genes and gene clusters implicated in lint fiber development. Genetics 176:2577-2588

Rong F, Tang L, Tang Y, Li Z, Wei Y (2015) Analysis of QTL mapping for fiber quality traits in introgression lines cotton. Molecular Plant Breeding 13:1509-1516

Said JI, Song M, Wang H, Lin Z, Zhang X, Fang DD, Zhang J (2015) A comparative meta-analysis of QTL between intraspecific Gossypium hirsutum and interspecific $G$. hirsutum $\times G$. barbadense populations. Mol Genet Genomics 290:1003-1025

Shang L, Wang Y, Cai S, Wang X, Li Y, Abduweli A, Hua J (2016) Partial dominance, overdominance, epistasis and QTL by environment interactions contribute to heterosis in two Upland cotton hybrids. G3 6:499-507

Shi Y, Zhang B, Li J, Liu A, Yuan Y (2008) Cotton fiber quality traits were controlled mainly by maternal plant genotype. Hereditas (Beijing) 30:1466-1476

Shi Y, Li W, Li A, Ge R, Zhang B, Li J, Liu G, Li J, Liu A, Shang H, Gong J, Gong W, Yang Z, Tang F, Liu Z, Zhu W, Jiang J, Yu X, Wang T, Wang W, Chen T, Wang K, Zhang Z, Yuan Y (2015) Constructing a high-density linkage map for Gossypium hirsutum $\times$ Gossypium barbadense and identifying QTLs for lint percentage. J Integr Plant Biol 57:450-467

Shi Y, Zhang B, Liu A, Li W, Li J, Lu Q, Zhang Z, Li S, Gong W, Shang H, Gong J, Chen T, Ge Q, Wang T, Zhu H, Liu Z, Yuan Y (2016) Quantitative trait loci analysis of Verticillium wilt resistance in interspecific backcross populations of Gossypium hirsutum $\times$ Gossypium barbadense. BMC Genomics 17:877. https://doi. org/10.1186/s12864-016-3128-X

Song W, Wang M, Su W, Lu Q, Xiao X, Cai J, Zhang Z, Li S, Li P, Gong J, Gong W, Shang H, Liu A, Li J, Chen T, Ge Q, Shi Y, Yuan Y (2017) Genetic and phenotypic effects of chromosome segments introgressed from Gossypium barbadense into Gossypium hirsutum. PloS ONE 12(9):e0184882

Stelly DM, Saha S, Raska DA, Jenkins JN, McCarty JC, Gutierrez OA (2005) Registration of 17 Upland (Gossypium hirsutum) cotton germplasm lines disomic for different G.barbadense chromosome or arm substitutions. Crop Sci 45:2663-2665

Su J, Chen H, Deng F, Ma Q, Pang C, Wu M, Wang C, Yu S (2014) Establishment and application of PCR-SSR based method to 
quickly detect exogenous chromosome fragments in cotton. J Agric Biotechnol 22:632-641

Tyagi P, Gore MA, Bowman DT, Campbell BT, Udall JA, Kuraparthy V (2014) Genetic diversity and population structure in the US Upland cotton (Gossypium hirsutum L.). Theor Appl Genet 127:283-295

Ulloa M, Saha S, Jenkins JN, Meredith WR, McCarty JC, Stelly DM (2005) Chromosomal assignment of RFLP linkage groups harboring important QTLs on an intraspecific cotton (Gossypium hirsutum L.) joinmap. J Hered 96:132-144

Van Berloo R (2008) GGT 2.0: versatile software for visualization and analysis of genetic data. J Hered 99:232-236

Voorrips RE (2002) MapChart: software for the graphical presentation of linkage maps and QTLs. J Hered 93:77-78

Wan XY, Wan JM, Su CC, Wang CM, Shen WB, Li JM, Wang HL, Jiang L, Liu SJ, Chen LM, Yasui H, Yoshimura A (2004) QTL detection for eating quality of cooked rice in a population of chromosome segment substitution lines. Theor Appl Genet 110:71-79

Wan X, Weng J, Zhai H, Wang J, Lei C, Liu X, Guo T, Jiang L, Su N, Wan J (2008) Quantitative trait loci (QTL) analysis for rice grain width and fine mapping of an identified QTL allele $g w-5$ in a recombination hotspot region on chromosome 5. Genetics 179:2239-2252

Wang P, Ding Y, Lu Q, Guo W, Zhang T (2008) Development of Gossypium barbadense chromosome segment substitution lines in the genetic standard line TM-1 of Gossypium hirsutum. Chin Sci Bull 53:1512-1517

Wang PZ, Shi LF, Su L, Hu BM (2010) Quantitative trait loci for resistance against fusarium wilt based on three cotton $\mathrm{F}_{2}$ populations. Agric Sci China 9:1799-1806

Wang P, Zhu Y, Song X, Cao Z, Ding Y, Liu B, Zhu X, Wang S, Guo W, Zhang T (2012) Inheritance of long staple fiber quality traits of Gossypium barbadense in G.hirsutum background using CSILs. Theor Appl Genet 124:1415-1428

Wang F, Xu Z, Sun R, Gong Y, Liu G, Zhang J, Wang L, Zhang C, Fan S, Zhang J (2013) Genetic dissection of the introgressive genomic components from Gossypium barbadense L. that contribute to improved fiber quality in Gossypium hirsutum L. Mol Breed 32:547-562

Wang H, Huang C, Guo H, Li X, Zhao W, Dai B, Yan Z, Lin Z (2015) QTL mapping for fiber and yield traits in Upland cotton under multiple environments. PLoS ONE 10:e130742

Wang F, Zhang C, Liu G, Chen Y, Zhang J, Qiao Q, Yuan Z, Fan S, Zhang J (2016a) Phenotypic variation analysis and QTL mapping for cotton (Gossypium hirsutum L.) fiber quality grown in different cotton-producing regions. Euphytica 211:169-183

Wang H, Huang C, Zhao W, Dai B, Shen C, Zhang B, Li D, Lin Z (2016b) Identification of QTL for fiber quality and yield traits using two immortalized backcross populations in Upland cotton. PloS ONE 11:e0166970

Wang Y, Wang X, Li Z, Yang X, Zhang Y, Wu L, Wu J, Zhang G, Ma Z (2016c) Development of Pima cotton chromosome segment substitution lines with Gossypium hirsutum background. J Plant Genet Resour 17:114-119

Wu J, McCarty JC, Jenkins JN, Meredith WR (2010) Breeding potential of introgressions into Upland cotton: genetic effects and heterosis. Plant Breed 129:526-532

Yang Z, Li J, Li A, Zhang B, Liu G, Li J, Shi Y, Liu A, Jiang J, Wang T, Yuan Y (2009) Developing chromosome segment substitution lines(CSSLs) in cotton (Gossypium) using advanced backcross and MAS. Mol Plant Breed 7:233-241

Yang X, Zhou X, Wang X, Li Z, Zhang Y, Liu H, Wu L, Zhang G, Yan G, Ma Z (2015) Mapping QTL for cotton fiber quality traits using simple sequence repeat markers, conserved intron-scanning primers, and transcript-derived fragments. Euphytica 201:215-230
You S (2015) Using chromosome segment introgresion lines to fine mapping QTL of cotton fiber quality traits on the Chr.A2, Chr.A7, Chr.A8 and Chr.D5. [D], Nanjing Agricultural University cnki.net (S562, in Chinese)

Yu J, Yu S, Gore M, Wu M, Zhai H, Li X, Fan S, Song M, Zhang J (2013) Identification of quantitative trait loci across interspecific $\mathrm{F}_{2}, \mathrm{~F}_{2: 3}$ and testcross populations for agronomic and fiber traits in tetraploid cotton. Euphytica 191:375-389

Yu S, Fan S, Wang H, Wei H, Pang C (2016) Progresses in research on cotton high yield breeding in China. Sci Agric Sin 49:3465-3476

Zhai H, Gong W, Tan Y, Liu A, Song W, Li J, Deng Z, Kong L, Gong J, Shang H, Chen T, Ge Q, Shi Y, Yuan Y (2016) Identification of chromosome segment substitution lines of Gossypium barbadense introgressed in G. hirsutum and quantitative trait locus mapping for fiber quality and yield traits. PLoS ONE 11:e0159101

Zhang J, Yao ting WU, Guo WZ, Zhang TZ (2000) Fast screening of microsatellite markers in cotton with PAGE/silver staining. Acta Gossypii Sin 05:267-269+282

Zhang J, Wang S, Shi Y, Zhang G, Liu A, Li J, Ma Z, Yuan Y (2008) Molecular marker and QTL for yield-related traits in transgenic Bacillus thuringiensis (Bt) hybrid cotton. Cotton Sci 20:179-185

Zhang ZS, Hu MC, Zhang J, Liu DJ, Zheng J, Zhang K, Wang W, Wan Q (2009) Construction of a comprehensive PCR-based marker linkage map and QTL mapping for fiber quality traits in Upland cotton (Gossypium hirsutum L.). Mol Breed 24:49-61

Zhang J (2012) The evaluation and identifying QTL of fiber yield and quality traits of chromosome segment substitution lines $\left(\mathrm{BC}_{5} \mathrm{~F}_{3}\right.$, $\mathrm{BC}_{5} \mathrm{~F}_{3: 4}, \mathrm{BC}_{5} \mathrm{~F}_{3: 5}$ ) in Upland cotton. [D], Chinese Academy of Agricultural Sciences cnki.net (S562, in Chinese)

Zhang J, Yu J, Pei W, Li X, Said J, Song M, Sanogo S (2015a) Genetic analysis of Verticillium wilt resistance in a backcross inbred line population and a meta-analysis of quantitative trait loci for disease resistance in cotton. BMC Genomics. https://doi.org/10.1186/ s12864-015-1682-2

Zhang M, Chen Q, Su X, Geng H, Qu Y (2015b) QTL mapping for 5 fiber quality traits in cotton hybrid populations between Sea Island cotton and Upland cotton(Gossypium barbadense L. and Gossypium hirsutum L.). Acat Agric Boreali-Occidentalis Sin 24:64-71

Zhang Z, Li J, Muhammad J, Cai J, Jia F, Shi Y, Gong J, Shang H, Liu A, Chen T, Ge Q, Palanga KK, Lu Q, Deng X, Tan Y, Li W, Sun L, Gong W, Yuan Y (2015c) High resolution consensus mapping of quantitative trait loci for fiber strength, length and micronaire on chromosome 25 of the Upland cotton (Gossypium hirsutum L.). PLoS ONE 10:e0135430

Zhang S, Feng L, Xing L, Yang B, Gao X, Zhu X, Zhang T, Zhou B (2016a) New QTLs for lint percentage and boll weight mined in introgression lines from two feral landraces into Gossypium hirsutum acc TM-1. Plant Breed 135:90-101

Zhang SW, Zhu XF, Feng LC, Gao X, Yang B, Zhang TZ, Zhou BL (2016b) Mapping of fiber quality QTLs reveals useful variation and footprints of cotton domestication using introgression lines. Sci Rep 6:31954

Zhao F, Zhang G, Zeng R, Yang Z, Zhu H, Zhong B, Ling Y, He G (2009) Additive effects and epistasis effects of QTL for plant height and its components using single segment substitution lines (SSSLs) in rice. Acta Agron Sin 35:48-56

Publisher's Note Springer Nature remains neutral with regard to jurisdictional claims in published maps and institutional affiliations. 